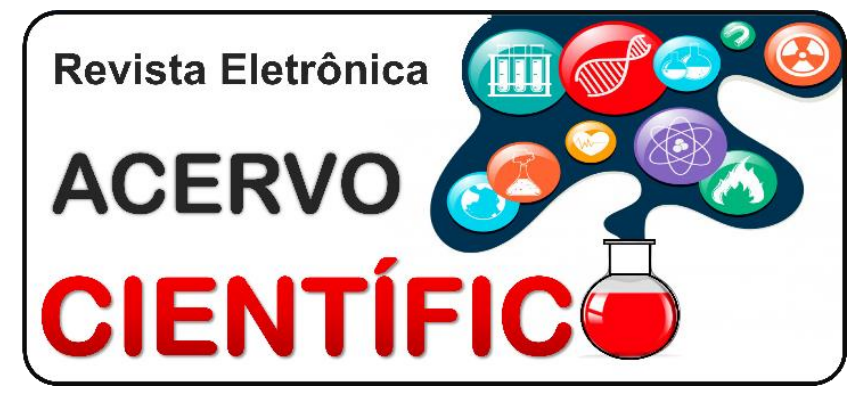

REVISÃO BIBLIOGRÁFICA

Recebido em: 7/2020

Aceito em: 8/2020

Publicado em: 1/2021

\title{
Gastrectomia com linfadenectomia a nível de D1 ou D2: uma revisão da literatura
}

\author{
Gastrectomy with lymphadenectomy at the level of D1 or D2: a review of the literature \\ Gastrectomía con linfadenectomía en los niveles D1 o D2: una revisión de la literatura
}

Tainara Sales Miranda ${ }^{1 *}$, Henrique Moura Parreira ${ }^{1}$, Arthur Leandro Ribeiro de Freitas ${ }^{2}$, Elisa Marques Franco ${ }^{1}$, Fernanda Pêgo Miranda Netto ${ }^{3}$, Gustavo Almeida Keller ${ }^{3}$, Kassielly Melissa Ribeiro Rodrigues ${ }^{4}$, Luíza Coimbra Teixeira ${ }^{2}$, Priscila Pereira Albuquerque ${ }^{4}$, Victor Martins Gonçalves ${ }^{3}$.

Resumo: Este artigo buscou analisar a produção científica sobre pacientes com câncer gástrico submetidos à gastrectomia com linfadenectomia $\mathrm{D} 2$, relacionando tal técnica com a redução da morbimortalidade $\mathrm{e}$ melhora do prognóstico. O câncer gástrico é uma das principais causas de mortalidade por câncer. A ressecção cirúrgica do tumor gástrico é tida como a única conduta terapêutica com possibilidade de cura. Nos países orientais, a dissecção linfonodal D2 é a técnica de escolha, as taxas de morbimortalidade são menores quando comparadas aos ocidentais que utilizam D1. Trata-se de uma revisão sobre pacientes com câncer gástrico submetidos à gastrectomia com linfadenectomia D2, buscando a relação desta técnica com a redução da morbimortalidade e melhora do prognóstico. A linfadenectomia D2 mostra-se mais positiva em relação à $D 1$. Foi observado complicações mais frequentes de fístulas pancreáticas no pós-operatório, em comparação à D1. A linfadenectomia D2, em consonância com a preservação do pâncreas e baço, se mostrou mais eficaz, proporcionando maior tempo de sobrevida aos pacientes que possuem tumores gástricos ressecáveis. Entretanto, variáveis como a experiência do cirurgião devem ser investigadas, a fim de melhorar o prognóstico e reduzir a morbimortalidade desses pacientes após as abordagens cirúrgicas.

Palavras-chave: Gastrectomia, Câncer de estômago, Excisão de linfonodo.

\begin{abstract}
This article sought to analyze the scientific production on gastric cancer patients undergoing gastrectomy with D2 lymphadenectomy, relating this technique to reducing morbidity and mortality and improving prognosis. Gastric cancer is a major cause of cancer mortality. Surgical resection of the gastric tumor is considered the only therapeutic approach with the possibility of cure. In eastern countries, D2 lymph node dissection is the technique of choice, the rates of morbidity and mortality are lower when compared to western patients using D1. This a review about that patients submitted to gastrectomy with D2 lymphadenectomy, looking for the relationship of this technique with the reduction of morbidity and mortality and improvement of the prognosis. D2 lymphadenectomy is more positive than D1. More frequent complications of pancreatic fistulas were observed in the postoperative period, compared to D1. D2 lymphadenectomy, in line with the preservation of the pancreas and spleen, proved to be more effective, providing longer survival times for patients who have resectable gastric tumors. However, variables such as the surgeon's experience should be investigated in order to improve the prognosis and reduce the morbidity and mortality of these patients after surgical approaches.
\end{abstract}

Keywords: Gastrectomy, Stomach neoplasms, Lymph node excision.

${ }^{1}$ Centro Universitário de Caratinga (UNEC), Caratinga - MG. *E-mail: tainarasmiranda@hotmail.com

2 Universidade Federal dos Vales do Jequitinhonha e Mucuri (UFVJM), Teófilo Otoni - MG.

3 Universidade Vale do Rio Doce (UNIVALE), Governador Valadares - MG.

${ }^{4}$ Pontifícia Universidade Católica de Minas Gerais (PUC - MG), Poços de Caldas - MG. 
Resumen: Este artículo buscó analizar la producción científica en pacientes con cáncer gástrico sometidos a gastrectomía con linfadenectomía D2, relacionando esta técnica con la reducción de la morbimortalidad y la mejora del pronóstico. El cáncer de estómago es una de las principales causas de mortalidad por cáncer. La resección quirúrgica del tumor gástrico se considera el único abordaje terapéutico con posibilidad de cura. En los países del este, la disección de los ganglios linfáticos D2 es la técnica de elección, las tasas de morbilidad y mortalidad son menores en comparación con los pacientes occidentales que utilizan D1. Es una revisión en pacientes con cáncer gástrico sometidos a gastrectomía con linfadenectomía D2, buscando la relación de esta técnica con la reducción de la morbimortalidad y la mejora del pronóstico. La linfadenectomía D2 es más positiva que la D1. Se observaron complicaciones más frecuentes de las fístulas pancreáticas en el postoperatorio, en comparación con D1. La linfadenectomía D2, en línea con la preservación del páncreas y el bazo, demostró ser más efectiva, proporcionando tiempos de supervivencia más largos para los pacientes con tumores gástricos resecables. Sin embargo, deben investigarse variables como la experiencia del cirujano para mejorar el pronóstico y reducir la morbimortalidad de estos pacientes tras los abordajes quirúrgicos.

Palabras clave: Gastrectomia, Neoplasias gástricas, Escisión del ganglio linfático.

\section{INTRODUÇÃO}

O adenocarcinoma é a neoplasia maligna de estômago mais comum e sua principal etiologia relacionase com a infecção da bactéria Helicobacter pylori, porém, existem outros fatores, hereditários e não hereditários, envolvidos em sua patogênese. O prognóstico e o tratamento são pautados de acordo com a localização e estadiamento do tumor, a existência de metástases e número de linfonodos acometidos e ressecados (BRASIL, 2017).

Corresponde à quinta neoplasia mais incidente e a terceira causa de mortalidade por câncer no mundo (OMS, 2018). No Brasil, há consonância com os dados mundiais, sendo essa incidência maior na população masculina, que apresentou 9.207 óbitos em contrapartida aos 5.107 óbitos na população feminina no ano de 2017 (INCA, 2020). A ressecção cirúrgica do tumor gástrico é tida como a única conduta terapêutica com possibilidade de cura. Contudo, há controvérsias entre as diferentes técnicas existentes, principalmente no que tange à extensão da dissecção linfonodal (LAM S, 2018).

Nos países orientais, o índice de mortalidade pós gastrectomia varia de 0,8 a $13 \%$, entretanto, os países ocidentais apresentam taxas maiores (TALAIEZADEH AH, et al., 2015). A escolha padrão por linfadenectomia D2 pelos cirurgiões orientais em oposição às dissecções mais limitadas no ocidente, pode ser uma hipótese para explicar essa diferença estatística (ÖZER I, 2017). A primeira técnica consiste na ressecção das cadeias linfonodais $\mathrm{N} 1$ e $\mathrm{N} 2$ e dos linfonodos proximais das artérias gástrica esquerda, hepática comum, esplênica e do tronco celíaco, enquanto a segunda, denominada linfadenectomia D1, é menos extensa, abrangendo apenas os linfonodos distantes até 3 centímetros do tumor primário (LOPASSO FP, et al., 2002).

O objetivo do presente estudo consiste em analisar a produção científica sobre pacientes com câncer gástrico submetidos à cirurgia de gastrectomia com linfadenectomia D2, buscando a relação desta técnica com a redução da morbimortalidade e melhora do prognóstico. Além disso, visa comparar estes resultados aos da técnica vigente no mundo ocidental, isto é, a linfadenectomia D1.

\section{REVISÃO BIBLIOGRÁFICA}

\section{Conceito}

O câncer gástrico (ou câncer de estômago) pode ser definido como o crescimento anormal de células no estômago. O tipo histológico mais comum é o adenocarcinoma, que corresponde a mais de $90 \%$ de casos de neoplasia maligna. Esse tipo de tumor geralmente ocorre na camada de revestimento interno, a chamada camada mucosa, que inicia o processo neoplásico com formações irregulares, como pequenas lesões com ulcerações. $O$ adenocarcinoma de estômago pode ser subdividido em dois tipos: o tipo intestinal e o tipo difuso de Lauren. O primeiro trata-se de um tumor mais diferenciado e evolui de lesões pré- 
malignas, enquanto o difuso de Lauren apresenta-se com padrão infiltrativo, tem extensão submucosa e metástases precoces, portanto, apresenta um prognóstico pior (BRASIL, 2017).

O câncer de estômago é o quarto tipo de câncer mais comum no mundo e, ainda, é a segunda maior causa de mortalidade (TAN Z, 2019). Já no Brasil, trata-se do quinto câncer mais frequente. Pode-se afirmar que há fatores hereditários e não hereditários associados ao aparecimento e desenvolvimento do câncer de estômago, ainda que a etiologia não seja muito conhecida. Determinadas situações são fatores de risco, a saber: a infecção gástrica pelo Helicobacter pylori, história familiar de polipose adenomatosa familiar e o próprio câncer gástrico, idade avançada, gastrite atrófica crônica, anemia perniciosa, hábitos de vida como dieta pobre em vegetais, consumo excessivo de alimentos em conserva, dieta rica em sal e exposição ao tabagismo (BRASIL, 2017).

No intuito de detectar precocemente o câncer gástrico, pode-se lançar mão de estratégias como o rastreamento e o diagnóstico precoce. A primeira estratégia é direcionada a determinado grupo populacional assintomático que irá obter mais benefícios por esta prática, bem como será mais beneficiada pela redução de mortalidade, por meio de aplicação de testes ou exames. Para o rastreamento de câncer gástrico, não há evidência científica que comprove que haja mais benefícios do que riscos, portanto não é recomendado. A outra estratégia, o diagnóstico precoce, se vale da redução do estágio de apresentação do câncer e, para isso, deve-se investigar os sinais e sintomas mais comuns: epigastralgia; dispepsia e disfagia; massa em abdômen superior; refluxo; perda de apetite e perda de peso ponderal (INCA, 2018).

O diagnóstico do câncer de estômago é realizado por meio de endoscopia digestiva alta (EDA) com biópsia e, em alguns casos, faz-se necessário o exame histoquímico para diferenciar adenocarcinomas pouco diferenciados e linfomas. Para o estadiamento do tumor, a tomografia computadorizada (TC), a videolaparoscopia e o PET-TC podem ser úteis (INCA, 2018).

O estadiamento do câncer gástrico pode ser obtido pela classificação TNM, preconizada pela União Internacional para o Controle do Câncer (UICC), que é idêntica à classificação da 15a edição do Japanese Classification of Gastric Carcinoma (JAPANESE GASTRIC CANCER TREATMENT GUIDELINES, 2018).

É importante realizar o estadiamento da doença, uma vez que se pode prever a taxa de sobrevida, analisar a taxa de crescimento do tumor, o tipo de câncer acometido, o tamanho tumoral e se há metástases à distância. $\mathrm{O}$ estadiamento pode ser clínico ou patológico. $\mathrm{O}$ clínico se dá mediante exame físico e análise dos exames complementares. O patológico se dá por achados cirúrgicos e através do exame anatomopatológico, o que revela a extensão do câncer com maior precisão (Quadro 1) (INCA, 2019).

O câncer de estômago tem seu prognóstico dependente da localização e estadiamento do tumor, número de linfonodos ressecados e acometidos, e presença de metástase. Pode ocorrer no órgão como um todo, localizar em sua porção proximal ou distal, junto ao piloro. Quanto à parte proximal, pode ou não afetar a junção gastroesofágica. Vários estudos mostram que mais da metade dos pacientes acometidos pelo câncer inicial na parte distal, podem ser curados quando o tumor for ressecado. Já os pacientes com tumores na porção proximal, podem ser curados em menos de $20 \%$ das vezes, por mais que estejam em fase inicial. É fato que a identificação dos fatores de risco, da doença em estágio inicial e o atendimento adequado, são de suma importância para um resultado terapêutico e consequente melhor prognóstico (BRASIL, 2017).

O tratamento cirúrgico com intenção de cura é o mais efetivo. Entretanto, a cirurgia só é indicada de acordo com o estadiamento. Quando o câncer é precoce e se limita às camadas mucosa e submucosa, há a possibilidade de tratamento por ressecção endoscópica - geralmente para lesões menores de $2 \mathrm{~cm}$, bem diferenciadas, não ulceradas, restritas à mucosa e sem invasão angiolinfática, ou por gastrectomia D1. Já a gastrectomia com linfadenectomia D2, no Brasil, é indicada para lesões avançadas e são feitas em (neo) adjuvância, com quimioterapia e, eventualmente, com radioterapia (ANDREOLLO NA, et al., 2019; INCA, 2018). O tipo de linfadenectomia ideal é intensamente discutido em todo o mundo e divide opiniões, principalmente entre os hemisférios oriental e ocidental. 
Quadro 1 - Estadiamento TNM do câncer gástrico, São Paulo, 2015.

\begin{tabular}{|c|c|c|c|c|c|}
\hline Estádio & Descrição & Estádio & Descrição & Estádio & Descrição \\
\hline TX & $\begin{array}{l}\text { Tumor primário não } \\
\text { pode ser avaliado }\end{array}$ & NX & $\begin{array}{l}\text { Linfonodos regionais } \\
\text { não podem ser } \\
\text { avaliados }\end{array}$ & MO & $\begin{array}{l}\text { Sem metástases } \\
\text { a distância }\end{array}$ \\
\hline TO & $\begin{array}{l}\text { Sem evidência de } \\
\text { tumor primário }\end{array}$ & No & $\begin{array}{l}\text { Sem envolvimento } \\
\text { linfonodal }\end{array}$ & M1 & $\begin{array}{l}\text { Com metástases } \\
\text { a distância }\end{array}$ \\
\hline Tis & $\begin{array}{l}\text { Carcinoma in situ. } \\
\text { Intraepitelial sem } \\
\text { invasão da lâmina } \\
\text { própria }\end{array}$ & N1 & $\begin{array}{l}\text { 1-2 linfonodos } \\
\text { invadidos }\end{array}$ & - & - \\
\hline T1 & $\begin{array}{c}\text { Invasão da lâmina } \\
\text { própria (muscular da } \\
\text { mucosa ou submucosa) }\end{array}$ & N2 & $\begin{array}{l}\text { 3-6 linfonodos } \\
\text { invadidos }\end{array}$ & - & - \\
\hline T1a & $\begin{array}{c}\text { Invasão da muscular da } \\
\text { mucosa }\end{array}$ & N3 & $\begin{array}{l}7 \text { linfonodos } \\
\text { invadidos }\end{array}$ & - & - \\
\hline T1b & Invasão da submucosa & N3a & $\begin{array}{l}7 \text { a } 15 \text { linfonodos } \\
\text { invadidos }\end{array}$ & - & - \\
\hline T2 & $\begin{array}{l}\text { Invasão da muscular } \\
\text { própria }\end{array}$ & N3b & $\begin{array}{l}>15 \text { linfonodos } \\
\text { invadidos }\end{array}$ & - & - \\
\hline T3 & $\begin{array}{c}\text { Penetração da } \\
\text { subserosa sem invasão } \\
\text { de peritônio visceral ou } \\
\text { órgãos adjacentes }\end{array}$ & - & - & - & - \\
\hline T4 & $\begin{array}{l}\text { Invasão da serosa ou } \\
\text { tecidos adjacentes }\end{array}$ & - & - & - & - \\
\hline T4a & Invasão da serosa & - & - & - & - \\
\hline T4b & $\begin{array}{l}\text { Invasão de órgãos } \\
\text { adjacentes }\end{array}$ & - & - & - & - \\
\hline
\end{tabular}

Fonte: Miranda TS, et al., 2020. Baseado em Barchi LC, 2015.

\section{Linfadenectomia}

A principal modalidade de tratamento para o câncer gástrico é a cirúrgica, e para que ela obtenha o resultado curativo, é necessário que haja também a linfadenectomia. A linfadenectomia consiste na retirada de grupos de linfonodos de diferentes extensões (D1, D1+ e D2), que são classificados de acordo com as posições anatômicas. A extensão da dissecção dos linfonodos é alvo de grandes debates envolvendo estudos e protocolos orientais e ocidentais. O propósito de tal discussão se deve à alta incidência de metastatização dos linfonodos, o que pode ocorrer no câncer gástrico, inclusive em estágios precoces. 0 risco de metástases em linfonodos cresce gradualmente conforme aumenta a profundidade da penetração tumoral, podendo alcançar taxas de $20 \%$ em adenocarcinomas com invasão de submucosa e mais de $50 \%$ dos casos pode metastatizar quando se trata de tumores T2 (ÖZER I, et al., 2017). Por essas razões, justifica-se a realização da dissecção linfonodal.

\section{Linfadenectomias D1 e D1+}

A linfadenectomia D1 é considerada a versão mais limitada da ressecção de linfonodos, por se tratar da retirada apenas do grupo 1 ou $\mathrm{N} 1$ dos linfonodos - os perigástricos -, que abrange as estações nos. 1-7 em 
gastrectomias totais (GT) e as estações nos. 1, 3, 4sb, 4d, 5, 6, 7 em gastrectomias distais (GD), de acordo com o último guideline japonês de tratamento do câncer gástrico. O guideline indica a linfadenectomia D1 para tumores de estadiamento cT1a que não entram em critérios para a ressecção endoscópica da mucosa (EMR) e para a dissecção endoscópica da submucosa (ESD). Também é indicada para tumores cT1bN0 que são tipicamente diferenciados histologicamente e com $1,5 \mathrm{~cm}$ de diâmetro ou menor. Já a linfadenectomia D1+ consiste na ressecção das estações de D1 mais a ressecção das estações nos. 8ª , 9, $11 \mathrm{p}$ para GT, e nas estações de D1 mais nos. 8a, 9 para GD. Este tipo de linfadenectomia é indicado para tumores cT1NO (Figura 1) (JAPANESE GASTRIC CANCER TREATMENT GUIDELINES, 2018).

\section{Linfadenectomia D2}

A linfadenectomia D2 é definida pela retirada cirúrgica de um ou mais grupos de linfonodos N1 e N2 que estão acoplados anatomicamente ao órgão ou lesão alvo e também às artérias mais próximas desses locais afetados (TONETO MG, et al., 2008). Além disso, a técnica consiste também na retirada do pâncreas e do baço durante a cirurgia, o que implica em uma piora dos resultados de recuperação dos pacientes, comparado aos procedimentos que evitam a remoção desses órgãos. A técnica é utilizada para a ressecção de adenocarcinomas gástricos, contendo ou não uma condição metastática e, geralmente, o procedimento é recomendado para pacientes com câncer gástrico curável (SONGUN I, et al., 2010).

As estações de linfonodos que estão sujeitas à linfadenectomia D2 são as do grupo D1 mais nos. 8a, 9, $11 \mathrm{p}, 11 \mathrm{~d}, 12 \mathrm{a}$ para GT. Já em GD, as estações linfonodais correspondem à D1 mais nos. 8a, 9, 11p, 12a. A indicação de ressecção mais extensa é para tumores potencialmente curáveis, como os de estadiamento cT2-T4, bem como os tumores cT1N. Assim como mencionado acima por Songun I, et al. (2010) e ratificado pelo guideline japonês de 2018, a esplenectomia deve ser evitada em GT de câncer avançado de estômago superior que não envolva a curvatura maior do órgão (Figura 1) (JAPANESE GASTRIC CANCER TREATMENT GUIDELINES, 2018).

Figura 1 - Dissecção de linfonodos em gastrectomia. Estações de linfonodos em azul necessitam ser dissecadas em dissecção D1. Além desses, estações com linfonodos em laranja devem ser dissecados em dissecção D1+ e estações de linfonodos em verde em dissecção D2.

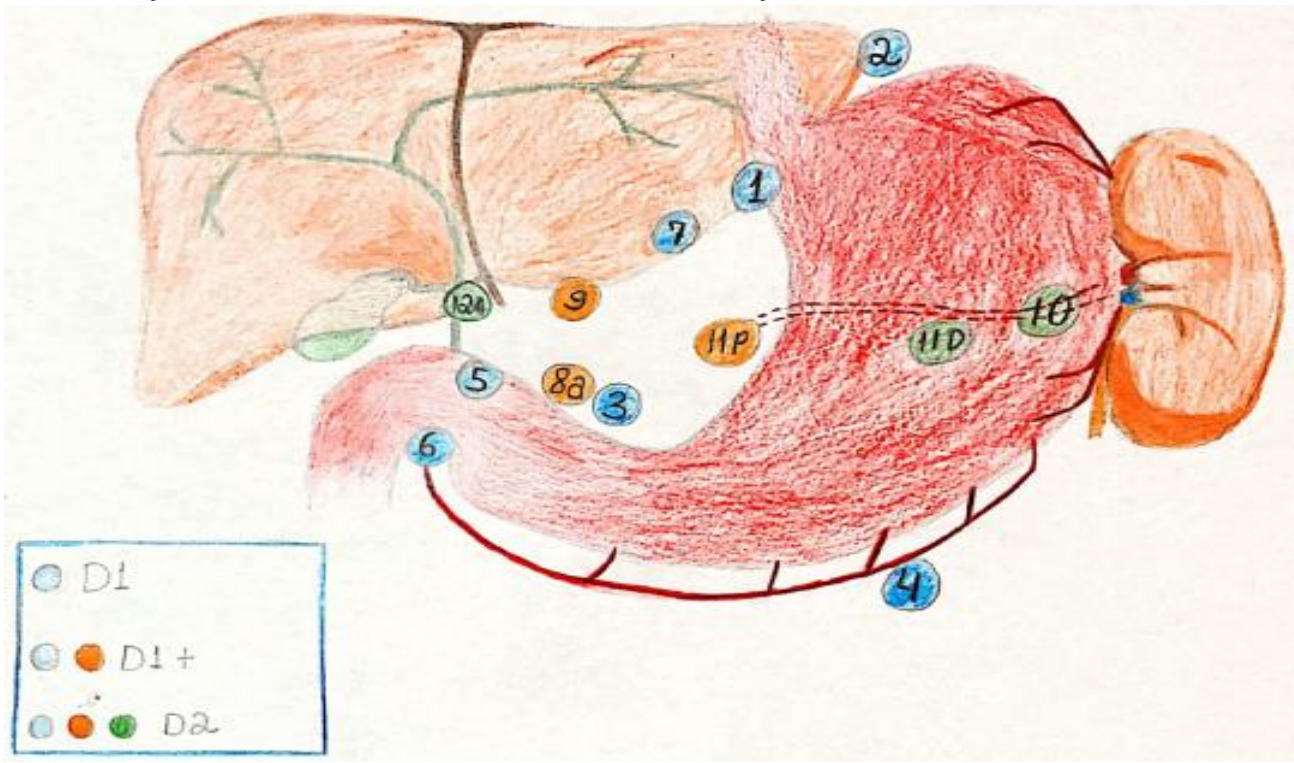

Fonte: Miranda TS, et al., 2020. Baseada em Japanese Gastric Cancer Treatment Guidelines, 2018. llustração cedida por Mariana Camilly Campos Miranda, 2021.

\section{Linfadenectomia D1 versus D2}

O tratamento do câncer gástrico suscita uma discussão importante no que tange à gastrectomia linfonodal D1 e D2. Alguns estudos demonstram diferentes resultados quanto à cirurgia de escolha para o tratamento do adenocarcinoma gástrico, principalmente nos países do ocidente que ainda não adequaram 
uma posição em relação à principal terapêutica de escolha para tratar o CA gástrico. Porém, existe maior consenso mundial de que a linfadenectomia D2 mostra-se mais positiva em relação à D1, principalmente evidenciado por estudos orientais que já aderem a gastrectomia D2 como principal tratamento para o adenocarcinoma (ÖZER I, et al., 2017).

No Brasil, as diretrizes diagnósticas e terapêuticas do adenocarcinoma de estômago, sugerem que a efetividade e segurança da linfadenectomia (mais extensa - D2 versus limitada - D1) é inconclusiva (BRASIL, 2017).

Muitos estudos recentes têm trazido novas comparações e resultados quanto às modalidades cirúrgicas. É evidenciado que a taxa de sobrevida após o procedimento cirúrgico de linfadenectomia D2 tem variação dependente da experiência do cirurgião, sendo um aumento da sobrevida global de 5 anos em pacientes que realizaram o procedimento com cirurgiões mais experientes. Ademais, foram observadas complicações mais frequentes de fístulas pancreáticas no pós-operatório, em comparação à cirurgia D1. No entanto, no total, apesar das complicações e experiência do profissional, a taxa de sobrevida da cirurgia D2 mostrou-se com melhores resultados comparadas à D1 (TUDOR S, et al., 2018).

Existem evidências em relação à mortalidade peri-operatória. Um importante estudo holandês datado da década de 90, teve como resultados mais precoces, maiores taxas de morbidade (43-46\%) e maiores taxas de mortalidade (10-13\%), sem conseguir comprovar algum benefício na sobrevida. Devido a esses resultados, que divergem da experiência oriental, o ensaio foi duramente criticado devido às variáveis a que estava sujeito, como a falta de experiência do cirurgião e das instituições participantes do estudo (MOGAL $\mathrm{H}$, et al., 2019). Apesar de todos esses pontos e vieses, este é o principal estudo feito no ocidente atualmente que discute a diferença entre as abordagens cirúrgicas D1 e D2 (YININ HU, et al., 2019).

A despeito dos resultados precoces, após 15 anos de seguimento do estudo, foi verificada que a sobrevida de pacientes submetidos à linfadenectomia mais extensa era maior que a dos pacientes submetidos à D1. Observaram, ainda, que a maioria das complicações pós-operatórias se dava quando era uma cirurgia conjunta a uma retirada de pâncreas e/ou de baço. Nesse sentido, foi atribuída à esplenectomia e à pancreatectomia a maior chance de complicações, bem como de aumento da morbimortalidade. Dessa forma, a linfadenectomia D2 foi observada como a melhor abordagem cirúrgica para aqueles pacientes que têm um tumor ressecável (YININ HU, et al., 2019).

Lam S, et al. (2018), em outro estudo, demonstraram parâmetros como estágio do tumor, localização do tumor, IMC e quimioterapia perioperatória como relevantes para influenciar na escolha da extensão da linfadenectomia utilizada. $\mathrm{O}$ aumento do IMC foi relacionado à maiores taxas de mortalidade de todos os cânceres quando comparados com pacientes de peso normal. No entanto, mesmo com influência desses parâmetros, os resultados mostram que a linfadenectomia D2 com preservação do baço e/ou pâncreas, não está associada a maus resultados a curto e a longo prazo comparadas à dissecção D1, que prediz uma sobrevida global baixa (SYMEONIDIS D, et al., 2019).

Estudos ocidentais também relataram melhora da sobrevida associada a uma gastrectomia D2 modificada, ou seja, com preservação do baço, de forma particular em câncer gástrico em estágio III. Este benefício de sobrevida pode ser observado também em uma análise de subgrupo de pacientes classificados N0 que foram submetidos a linfadenectomia D2. Dessa forma, mesmo tais parâmetros sendo necessários à determinação do tipo de linfadenectomia, eles não alteram os resultados que demonstram a superioridade do tipo D2 (SYMEONIDIS D, et al., 2019).

Ademais, existe também o risco de recorrência do câncer após o procedimento de linfadenectomia. Essa taxa de recorrência também foi demonstrada comparativamente entre os procedimentos cirúrgicos D1 e D2, na qual foi demonstrado que a linfadenectomia D2 mostrou-se com uma taxa de recorrência de 30\% enquanto a dissecção a nível D1 apresentou 41\%. Assim sendo, para o fim de redução da recorrência, a extensão da retirada de linfonodos mais indicada é a D2 (BARRETO SG, et al., 2017).

Barreto SG, et al. (2017), analisaram vários estudos no quesito aumento de sobrevida após uma cirurgia de gastrectomia, seja a nível D1 ou D2 e, de acordo com os dados levantados, para todos os estágios de 
câncer gástrico, a dissecção de até 40 linfonodos parece ser necessária. No entanto, após análise dos resultados clínicos e de uma conclusão lógica dos fatos, concluiu-se que a melhor alternativa para o tratamento do câncer gástrico seja a linfadenectomia D2.

\section{CONSIDERAÇÕES FINAIS}

A partir dos dados supracitados, a técnica de ressecção linfonodal mais extensa, denominada linfadenectomia D2, em consonância com a preservação do pâncreas e baço durante a cirurgia, se mostrou mais eficaz quando comparada à linfadenectomia D1, proporcionando maior tempo de sobrevida aos pacientes que possuem tumores gástricos ressecáveis. Entretanto, variáveis como a experiência do cirurgião devem ser investigadas, com o objetivo de melhorar o prognóstico e reduzir a morbimortalidade destes pacientes após as abordagens cirúrgicas.

\section{REFERÊNCIAS}

1. ANDREOLLO NA, et al. Adjuvant chemoradiotherapy after subtotal or total gastrectomy and d2 limphadenectomy increases survival in advanced gastric cancer? Arquivos Brasileiros de Cirurgia Digestiva, 2019; 32(4): 1464.

2. BARCHI LC. Previsão de recorrência do câncer gástrico após cirurgia potencialmente curativa: validação externa do nomograma preconizado pelo GIRCG (Grupo Italiano de Estudo do Câncer Gástrico). Dissertação (Mestrado em Gastroenterologia) - Faculdade de Medicina da USP. São Paulo, 2015.

3. BARRETO SG, SIROHI B. Why should we perform a D2 lymphadenectomy in gastric cancer? Future Oncology, 2017; 13(23): 2009-2012.

4. BRASIL. Ministério da Saúde. Diretrizes diagnósticas e terapêuticas adenocarcinoma de estômago. Brasília, DF, 2017.

5. INCA. 2020. In: Tipos de Câncer: câncer gástrico. Disponível em: https://www.inca.gov.br/tipos-de-cancer/cancerde-estomago. Acesso em: 01 de agosto de 2020.

6. INCA, 2018. Tipos de câncer, câncer de estômago - versão para profissionais de saúde. Disponível em: https://www.inca.gov.br/tipos-de-cancer/cancer-de-estomago/profissional-de-saude. Acesso em: 01 de agosto de 2020.

7. INCA, 2019. Estadiamento do câncer. Disponível em: https://www.inca.gov.br/estadiamento. Acesso em: 01 de agosto de 2020.

8. GASTRIC CANCER. Japanese gastric cancer treatment guidelines. $5^{\text {th }}$ ed. Japan, 2020.

9. LAM S, et al. A comparison of the operative outcomes of D1 and D2 gastrectomy performed at a single Western center with multiple surgeons: a retrospective analysis with propensity score matching. World Journal of Surgical Oncology, 2018; 16:136.

10. LOPASSO FP, et al. Câncer Gástrico: linfadenectomia, técnica, padronização e resultados. Revista da Associação Médica Brasileira, 2002; 52(4): 189-212.

11. MOGAL H, et al. In Patients with Localized and Resectable Gastric Cancer, What is the Optimal Extent of Lymph Node Dissection-D1 Versus D2 Versus D3? Annals of Surgical Oncology, 2019; 26(9): 2912-2932.

12. OMS. 2018. In: OPAS Brasil: folha informativa câncer. Disponível em: https://www.paho.org/bra/index.php?option=com_content\&view=article\&id=5588:folha-informativacancer\&ltemid=1094. Acesso em: 01 de agosto de 2020.

13. ÖZER I, et al. Tendências em Cirurgia de Câncer Gástrico. Balcãs Med J., 2017; 34(1): 10-20.

14. SONGUN I, et al. Surgical treatment of gastric cancer: 15-year follow-up results of the randomised nationwide Dutch D1D2 trial. Lancet Oncol., 2010; 11(5): 439-449.

15. SYMEONIDIS D, et al. Current role of lymphadenectomy in gastric cancer surgery. Journal of BU ON.: Official Journal of the Balkan Union of Oncology, 2019; 24(5): 1761-1767.

16. TALAIEZADEH AH, et al. Mortality and Morbidity and Disease-Free Survival after D1 and D2 Gastrectomy for Stomach Adenocarcinomas. Asian Pac J Cancer Prev, 2015; 16(13): 5253-5256.

17. TAN ZHAOYANG. Recent Advances in the Surgical Treatment of Advanced Gastric Cancer: A Review. Medical Science Monitor, 2019; 25: 3537-3541.

18. TONETO MG, et al. Linfadenectomia ampliada (D2) no tratamento do carcinoma gástrico: análise das complicações pós-operatórias. Revista do Colégio Brasileiro de Cirurgiões, 2008; 35(4): 229-234.

19. TUDOR S, et al. D2 Lymphadenectomy for Gastric Adenocarcinoma: Long-term Results and the Impact of Surgeon Experience on the Survival Rates. Chirurgia, 2018; 113: 772-779.

20. YININ HU, et al. Comparative Effectiveness of Lymphadenectomy Strategies During Curative Resection for Gastric Adenocarcinoma. Journal of Gastrointestinal Surgery, 2019. 